\title{
periferio
}

\section{POLÍTICAS PÚBLICAS PARA LA EDUCACIÓN SECUNDARIA: ENTRE LA OBLIGATORIEDAD Y LA INCLUSIÓN}

\author{
Camila Carlachiani ${ }^{1}$ \\ Universidad Nacional de Rosario
}

\section{Resumen}

La educación secundaria resuena como uno de los nudos problemáticos del sistema educativo argentino y también de la región. Con una impronta eminentemente elitista de carácter enciclopédico, su desarrollo y expansión a través del tiempo, llegan a hoy a dislocar su propia marca de origen. Así, la promoción de inéditas políticas educativas para este nivel, se convierte en el principal eje de análisis de este trabajo. A través de la aproximación a un estudio comparado, se analizan las políticas educativas desarrolladas en Argentina, Uruguay, y Chile en los últimos diez años para la educación secundaria. La elección de estos países se debe a que fueron pioneros en la extensión total de su obligatoriedad en el territorio latinoamericano. Los Planes de Mejora Institucional (PMI) en Argentina; el Programa de Impulso a la Universalización (PIU) en Uruguay y el Liceo para Todos en Chile se constituyen en objetos de estudio vislumbrando aspectos comunes en las tres políticas analizadas a la vez que se sitúan las particularidades que otorgan singulares improntas en cada país, y en cada institución. La tensión entre lo local y lo global (BALL, 2001) articula el eje de análisis. La perspectiva de la Educación Comparada como metodología de investigación permite describir un fenómeno en diferentes contextos, analizarlo, visibilizar similitudes, diferencias, construcciones diversas. La Educación Comparada despliega una serie de metodologías y enfoques diferentes para desarrollar formas innovadoras de afrontar problemas complejos y analizar creativamente los datos educativos en un marco transcultural. (ALTBACH; KELLY, 1990).

Palabras clave: educación secundaria; políticas educativas; local/global; obligatoriedad; inclusión

\footnotetext{
${ }^{1}$ Prof. y Lic. en Ciencias de la Educación (UNR). Profesora Adjunta de la Cátedra Pedagogía Institucional del Profesorado Universitario y la carrera de Psicopedagogía (UAI). Miembro del Centro de Estudios e Investigaciones sobre el Curriculum y la Didáctica de la Facultad de Humanidades y Artes (UNR). camilacarlachiani@gmail.com
} 


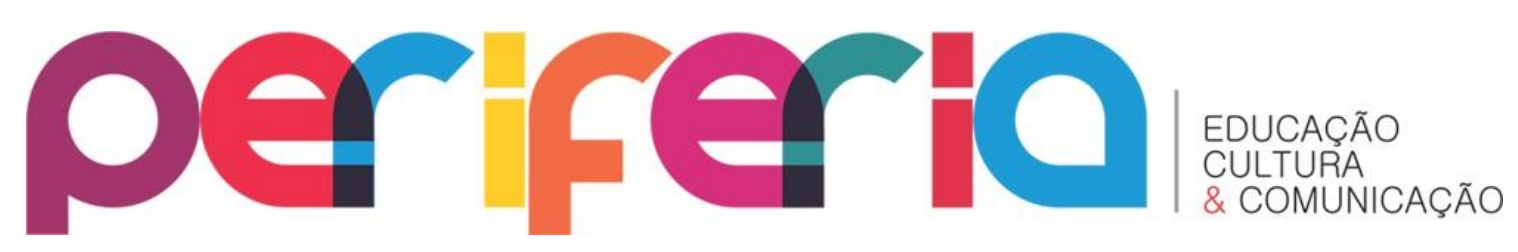

\title{
PUBLIC POLICIES FOR SECONDARY EDUCATION: BETWEEN OBLIGATORINESS AND INCLUSION
}

\begin{abstract}
Secondary education resound as one of the problematic nodes of the educational system, in Argentina and also in the region. With an eminently elitist imprint of an encyclopedic character, its development and expansion over time, come to dislocate its own brand of origin. Thus, the elaboration of new regulations that promote unpublished public policies for this level, becomes the main axis of analysis of this work. Through the approach to a comparative study, the educational policies developed in Argentina, Uruguay, and Chile in the last ten years for secondary education are analyzed. The choice of these countries is due to the fact that they were pioneers in the total extension of their obligatoriness in the Latin American territory. Institutional Improvement Plans (PMI) in Argentina; The Program for the Promotion of Universalization (PIU) in Uruguay and the Lyceum for All in Chile, constitute objects of study with a view to common aspects in the three policies analyzed, as well as the peculiarities that provide singular imprints in each country and in each institution. The tension between the local and the global (BALL, 2001) articulates the axis of analysis. The perspective of Comparative Education as a research methodology is a valuable tool that allows not only to describe a phenomenon in different contexts but also to analyze it, to visualize similarities, differences, different constructions. As Altbach and Kelly (1990) argue, Comparative Education deploys a number of different methodologies and approaches to develop innovative ways of addressing complex problems and creatively analyzing educational data in a cross-cultural framework.
\end{abstract}

Keywords: secondary education; educational policies; local/global; obligatoriness; inclusion 


\section{periferio}

\section{NUEVOS IMPERATIVOS PARA LA EDUCACIÓN SECUNDARIA}

Hace tiempo que el mandato escolar moderno de la escuela secundaria con su impronta eminentemente elitista y propedéutica está cambiando sus sentidos. En un contexto social cuyos avances tecnológicos y científicos se dan a pasos agigantados, donde las culturas y las economías regionales se globalizan, las noticias recorren el mundo a la velocidad de la luz y las políticas trascienden a los Estados Nacionales, la educación secundaria encuentra dislocado su objetivo de origen.

Siguiendo a Benavot (2006) la tradicional naturaleza elitista de la escuela secundaria se ha transformado en la medida que los países aplican políticas de acceso abierto, alcance universal y establecen programas que ofrecen asignaturas curriculares más amplias. Entre los principales cambios que afectan a dicha transformación, el autor menciona: la ampliación de los propósitos y objetivos de la educación secundaria; su diferenciación en ciclo básico y superior; el establecimiento de nuevos mecanismos de selección para facilitar su transición desde la educación primaria; y la diversificación de programas y ofertas curriculares para atender a las necesidades e intereses de las poblaciones heterogéneas de estudiantes en aumento. En este sentido, progresivamente se extendieron los años de obligatoriedad de este nivel.

En América Latina, los años ' 90 significaron un gran vaciamiento de los sistemas educativos debido a la instalación de políticas educativas neoliberales. Así, a la vez que los sistemas se descentralizaban, las escuelas recibían a grupos de estudiantes cada vez más heterogéneos para quienes la escuela secundaria no fue pensada ni diseñada. De acuerdo con Gajardo (1999) por su amplitud y extensión sistemática estas transformaciones fueron categorizadas como "reformas educativas" y correspondieron a una tendencia más general que ha sido identificada como una "ola de reformas" que se extendió por todo el subcontinente latinoamericano. Sin embargo, esta expansión fue de la mano de una importante expulsión. Felicitas Acosta (2012) señala que la escuela secundaria: 


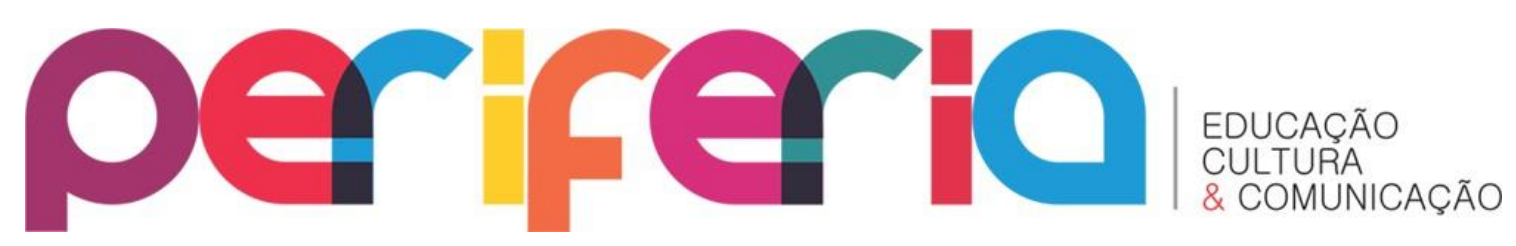

Es producto del cruce de dos tendencias frente a la expansión: la continuidad del modelo institucional tradicional que generó condiciones para la expansión-incorporación de matrícula constante -y la expulsión- alto índice de desgranamiento ( $\mathrm{p}$. 136).

En este sentido, Claudia Jacinto (2009) plantea la crisis de la escuela secundaria a partir de que su expansión en torno a la extensión de la obligatoriedad (la cual produjo la llegada de nuevos sectores sociales) eclosiona con su matriz selectiva cuyo curriculum es de carácter academicista. Por ello, es que algunos autores afirman que este proceso de ampliación del derecho y el establecimiento de la obligatoriedad de la educación secundaria, no se dio sin contradicciones. Tal como sostienen Pineau y Southwell (2010):

Paradójicamente esta voluntad de universalizar el nivel medio se da simultáneamente con un cuestionamiento de la institución escolar en general y de la escuela media en particular, como dispositivo de transmisión cultural y de integración de una población socio-cultural heterogénea. (PINEAU; SUTHWELL, 2010, p. 4).

Se presenta entonces un escenario social complejo donde las dimensiones políticas, económicas, culturales, entran en juego a la hora de pensar el sentido, o bien, los sentidos de la escuela secundaria actual. Es importante señalar que este proceso no se desarrolla en el vacío, sino que hay una malla política y social ligada a nuevas concepciones en torno al derecho a la educación que a partir de los '2000 permite un viraje de las políticas públicas, de la mano de gobiernos de centro izquierda en países de América Latina. Este viraje se vincula fuertemente a los lineamientos que a nivel mundial los diferentes países establecen a través la UNESCO (Organización de las Naciones Unidad para la Educación, la Ciencia y la Cultura -United Nations Educational, Scientific and Cultural Organization) para el desarrollo de la 


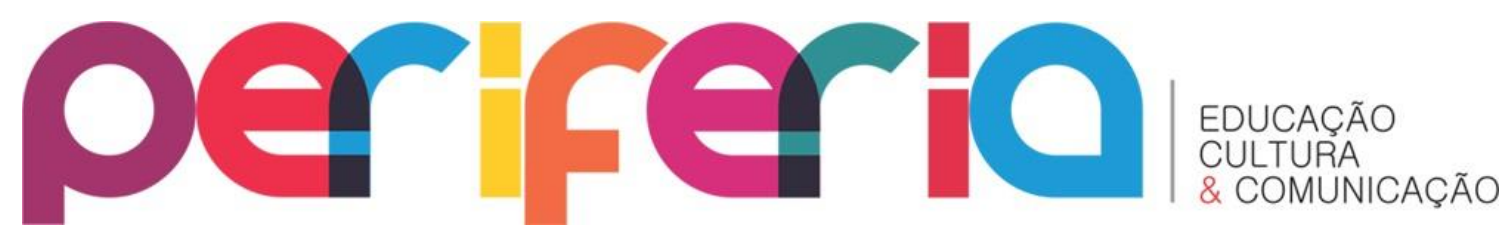

educación. Así, en el año 2000 los países del mundo se comprometieron en desarrollar esfuerzos sostenidos para la Educación Para Todos (ETP).

Los acuerdos sobre la necesidad de una agenda global común, que diera un impulso inédito a la educación comenzaron en 1990 con la Declaración Mundial de la Educación para Todos en Jomtien, Tailandia. En el 2000, en Dakar, Senegal, se construyó un marco de acción estratégico y se establecieron seis objetivos concretos a lograr en quince años (...) educación y cuidado de la primera infancia, la educación primaria universal, el aprendizaje de jóvenes y adultos, la alfabetización, la paridad de género y la calidad de la educación. La UNESCO coordina y lidera los esfuerzos internacionales para contribuir a estos objetivos, monitorea los avances y fomenta acciones a nivel global, regional y nacional hacia su logro (Oficina Regional de Educación para América Latina y El Caribe, UNESCO, 2013, p. 7).

Cabe destacar en este punto, las tensiones que se desarrollan en torno a lo local y lo global (BALL, 2001 apud CASIMIRO LOPES et. al. 2013) donde las políticas nacionales necesitan ser comprendidas como producto de un nexo de influencias e interdependencias que resultan de una interconexión, multiplicidad, hibridación; esto es, una combinación de lógicas globales, distantes y locales. De esta manera, se produce un juego de traducciones (CASIMIRO LOPES et. al., 2013) entre los diferentes niveles de decisión de las políticas educativas, desde el supra hasta el nano nivel. El supra nivel se compone de las políticas educativas y curriculares que emanan de organismos internacionales como pueden ser la UNESCO, el Banco Mundial, la OCDE, etc. Se trata de organizaciones que superan los límites de los Estados y diseñan políticas a nivel mundial para la educación. Luego, el macro nivel, corresponde a los ámbitos de diseño y decisión política en el marco de cada Estado Nacional o país. El meso nivel se vincula a las jurisdicciones que dentro de un Estado organizan políticamente el territorio. En Argentina, por ejemplo, el meso nivel corresponde a las jurisdicciones provinciales. Siguiendo este recorrido del nivel supra al nano, encontramos el nivel micro, que se refiere a las decisiones y acciones que se llevan adelante al interior de las instituciones 


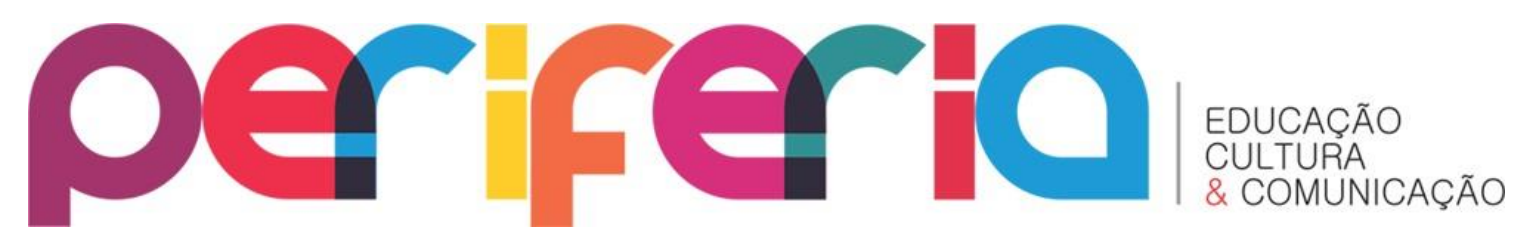

educativas. Por último, en el nivel nano, nos referimos al aula, como el ámbito concreto en el que las decisiones políticas se materializan a través del trabajo concreto de los profesores (STIRLING NETWORK FOR CURRICULUM STUDIES, 2016).

Volviendo al punto de las políticas educativas para la educación secundaria en América Latina, en un primer momento, en el supra nivel se desarrollaron primero acciones focalizadas que funcionaron como un remedio parcial ante las crecientes desigualdades, y luego universalistas planteadas como derechos ciudadanos. De este modo, la transformación más importante de las últimas décadas en la educación secundaria ha consistido en un cambio respecto de su definición viéndosela ahora como parte constitutiva de la educación fundamental que todo ciudadano debiera poseer, y ya no como una situación excepcional o del privilegio (Oficina Regional de Educación para América Latina y El Caribe, UNESCO, 2013). Ante este panorama Bentancur (2007) sostiene que:

[...] luego de algunos años de desarrollo, evaluación y crítica de los cambios procesados en los noventa, los gobiernos de Argentina, Chile y Uruguay han prohijado recientemente revisiones de dichas reformas, involucrando en la discusión, con distintos formatos, a múltiples actores políticos y sociales. (p. 161).

Es a partir de este proceso que dichos países han desarrollado nuevas políticas y normativas para la educación en general y la escuela secundaria en particular. Al interior de las escuelas se despliegan innumerables experiencias que asumen el desafío de transformar el formato escolar tradicional de la escuela secundaria con el fin de lograr la real inclusión de los jóvenes (para quienes la escuela secundaria no fue pensada), esto es: el ingreso, la permanencia, el aprendizaje y el egreso en tiempo y forma.

Para referirse al formato escolar de la escuela secundaria como ese núcleo difícil de modificar a pesar de los cambios y transformaciones que se dan a nivel social, político, económico y cultural, Terigi (2008) elabora la 


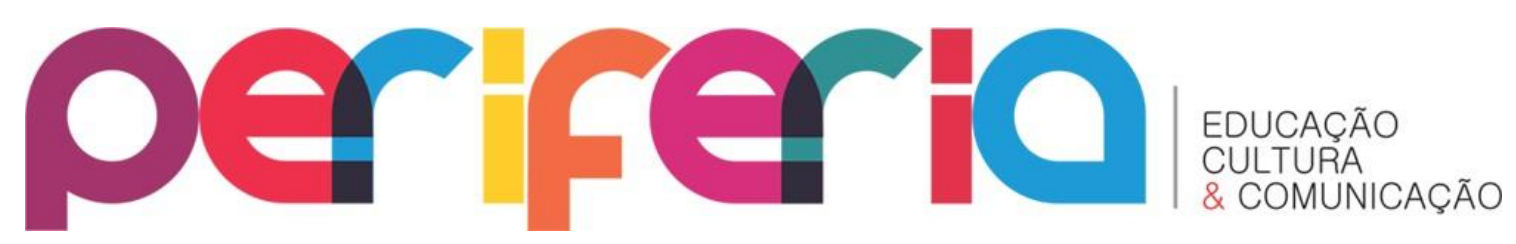

noción del trípode de hierro. Esta categoría ilustra la fortaleza casi inamovible de tres elementos clave que operan en la dinámica de la escuela secundaria, y, muchas veces, se presentan como obstáculos a la hora de pensar en generar transformaciones en su interior. En palabras de la autora: “la escuela media se estructuró en torno a tres disposiciones básicas: la clasificación de los currículos, el principio de designación de profesores por especialidad y la organización del trabajo docente por horas de clase" (TERIGI, 2008, p. 64). Se trata de un patrón organizacional que es difícil de modificar, aunque no se trata de una tarea imposible. En este sentido, Acosta (2012) presenta algunas estrategias en pos de superar el modelo excluyente de la escuela secundaria: a). dentro de la oferta regular; b) a través de modelos alternativos de escolarización; c). a través de la revalorización de la orientación para el trabajo. De esta manera, el tipo de cambio que se requiere en el nivel secundario involucra una transformación profunda en el formato escolar que asumió históricamente el nivel (TERIGI, 2008, p. 69).

Uno de los modos a través de los cuáles sería posible ofrecer dispositivos que otorguen nuevos sentidos al estar, habitar, transitar la escuela secundaria, es instaurar en la enseñanza el criterio de contemporaneidad (GUREVICH, 2009). Se trata de ofrecer a los estudiantes tramas de sentido entre las vidas individuales y el contexto de la época teniendo en cuenta la materialidad de los nuevos recursos y objetos tecnológicos; y las subjetividades particulares que se producen a través de los múltiples símbolos y representaciones presentes en la vida cotidiana de nuestros días. En palabras de la autora:

Si pensamos las culturas en plural, los territorios en plural, las sociedades en plural, hemos de transmitir a los estudiantes múltiples y diversas lógicas, preferencias y puntos de vista de los sujetos individuales y colectivos que permanentemente modelan y construyen las diferentes textualidades del mundo (GUREVICH, 2009, p. 25). 


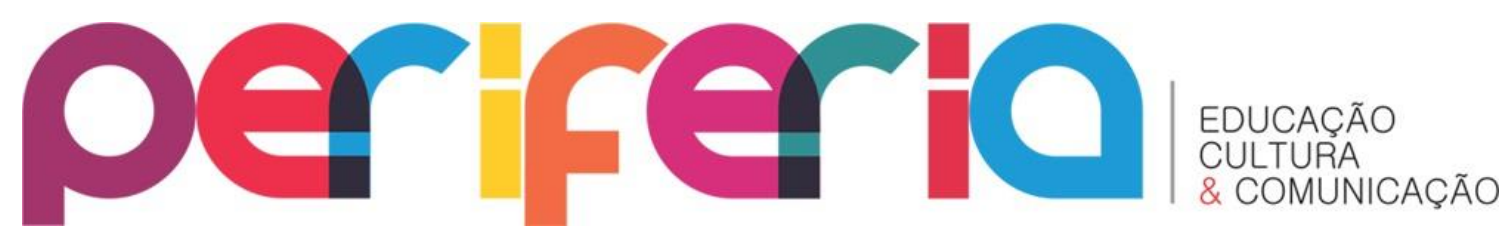

En relación a esto, acudimos a los aportes de Sánchez Tepatzi (2011), quien analiza los significados de valor que los estudiantes asignan a la escuela secundaria mediante la experiencia escolar. Para ello, su trabajo se centra en estudiar cómo se traduce el curriculum formal en una experiencia escolar relevante y cómo influye tal significado en la permanencia escolar. También resignifica ciertos indicadores tradicionales postulando que el abandono escolar, la reprobación y la eficiencia terminal no son indicadores que dan cuenta de la calidad educativa puesto que no ofrecen elementos que expliquen el dominio de los conocimientos. Debido a ello se los podría considerar como indicadores de la cobertura, porque sólo ofrecen información acerca del número de personas que ingresan, permanecen y egresan. En este sentido, la idea de experiencia escolar relevante y la resignificación de los indicadores son sumamente potentes para pensar y analizar la relación de los jóvenes con la escuela. Es posible vislumbrar, a través de los aportes mencionados, que la dimensión institucional en torno a lo que la escuela ofrece, parece ser un punto clave a la hora de garantizar la obligatoriedad y la inclusión en el nivel secundario.

Pinkasz (2015), postula que muchas de las políticas educativas desarrolladas en los últimos años para la educación secundaria se centran en la escuela como unidad de desarrollo del cambio. Esto significa que son los equipos directivos y docentes los que asumen la responsabilidad de la mejora escolar y de los aprendizajes ya que son ellos quienes conocen las necesidades de la comunidad. Sin embargo, la institución escolar no sólo se compone de directivos y docentes. Tal como sostiene Santos Guerra (2010) "la escuela es un proyecto compartido de la comunidad que se desarrolla en un contexto y un tiempo a través de la acción programada, intencional, y consensuada de todos sus miembros" (p.296). Para que este consenso sea posible, es necesario que existan cuatro códigos compartidos: código semántico, código ideológico, código ético y código de grados.

En América Latina, algunos ejemplos podrían ser los Planes de Mejora Institucional (PMI) en Argentina, el Programa de Impulso a la Universalización 


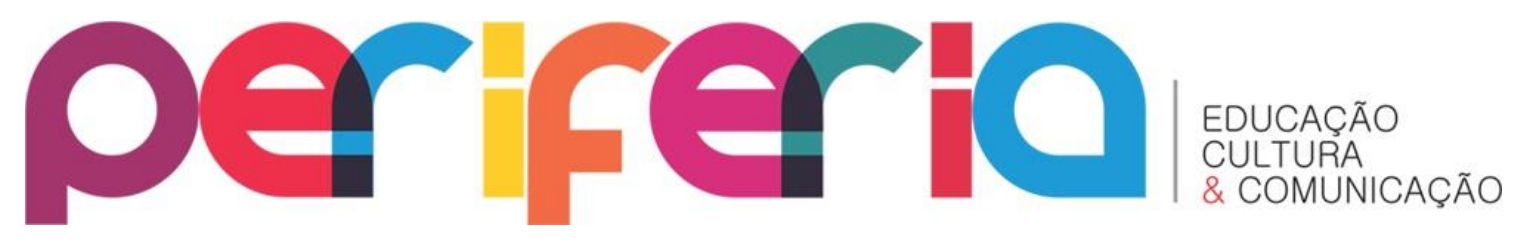

(PIU) en Uruguay, y Planes de Acción del Liceo para Todos en Chile. Esta generación de programas incorpora la idea de que la fuerza innovadora está en la escuela asumiendo las siguientes características: toman como unidad de cambio y análisis a la escuela; su propósito es producir una mejora sensible en los resultados educativos de un grupo de escuelas; orientan recursos adicionales a las escuelas bajo determinada política o programa; la escala puede ser nacional, territorial o de un grupo de escuelas; las escuelas objeto del programa pueden vincularse en red; las responsabilidades de diseño, conducción y supervisión pueden ser gubernamentales, sociales, privados o mixtos (PINKASZ, 2015). Ahora bien, cabe preguntarnos entonces ¿cómo se traducen estos programas emanados del macro nivel en el micro nivel institucional? ¿De qué modo se juega la tensión entre lo local y lo global?

\section{ARGENTINA: PLAN DE MEJORA INSTITUCIONAL (PMI)}

A partir del año 2006, con la sanción de la Ley de Educación Nacional 26.206 se establece la obligatoriedad del nivel secundario. Así, el art. 29 establece que: “la Educación Secundaria es obligatoria y constituye una unidad pedagógica y organizativa destinada a los/as adolescentes y jóvenes que hayan cumplido con el nivel de Educación Primaria”. Además, el art. 30 describe sus tres principales objetivos: "La Educación Secundaria en todas sus modalidades y orientaciones tiene la finalidad de habilitar a los/las adolescentes y jóvenes para el ejercicio pleno de la ciudadanía, para el trabajo y la continuación de estudios". Con este antecedente normativo, diferentes resoluciones del Consejo Federal de Educación (CFE) sintetizan y dan integralidad a las políticas con el fin de alcanzar el objetivo de la obligatoriedad: Resolución CFE 84/09 “Lineamientos Políticos y Estratégicos de la Educación Secundaria Obligatoria”; Resolución CFE 88/09 “Institucionalidad y Fortalecimiento de la Educación Secundaria Obligatoria: Planes Jurisdiccionales y Planes de Mejora Institucional (PMI)"; Resolución CFE 93/09 “Orientaciones para la organización pedagógica e institucional de la 


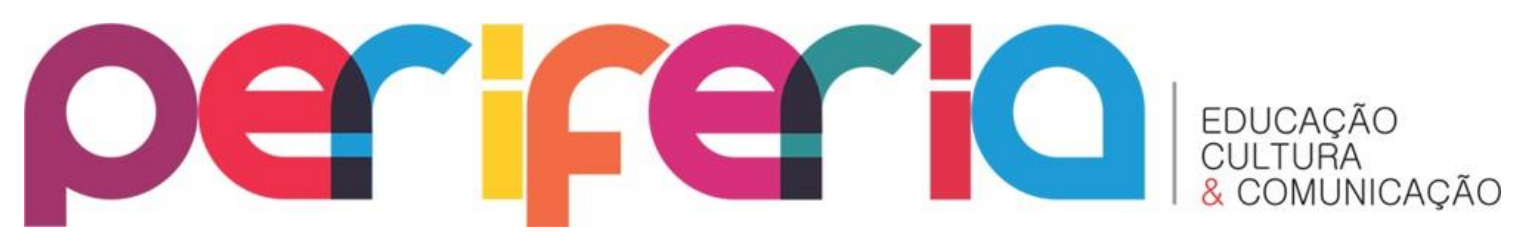

Escuela Secundaria Obligatoria"; Resolución CFE 103/10 "Pautas Federales para la movilidad estudiantil en la Educación Obligatoria"; Resolución CFE 102/10 "Propuestas de inclusión y/o regularización de trayectorias escolares en la educación secundaria". Dentro de este marco normativo, los PMI (Planes de Mejora Institucional) se convierten en un instrumento para que la propuesta educativa del nivel secundario se despliegue en múltiples concreciones con arraigo y sentido institucional.

Por otra parte, la Ley $\mathrm{N}^{\circ}$ 26.075/05 de Financiamiento Educativo fue otro instrumento poderoso para llevar a cabo las políticas descriptas por medio del cual la inversión que realizó el gobierno nacional aumentó tanto en términos absolutos como porcentuales (GOROSTIAGA, 2012).

Entre las principales políticas para la educación secundaria desarrolladas en Argentina en los últimos años cabe mencionar: los Planes de Mejora Institucional (PMI), Escuelas de Reingreso de la Ciudad de Buenos Aires, Plan FinEs, Centro de Actividades Juveniles (CAJ), entre otros. En el presente trabajo, por razones de extensión, sólo se abordarán las acciones vinculadas al desarrollo del PMI. Este programa consiste en una política pública nacional a través de la cual el Estado transfiere recursos a las provincias y a las escuelas para que instrumenten modificaciones al modelo tradicional de la escuela secundaria incorporando variaciones en su organización académica (PINKASZ, 2015) con el fin de acompañar a las trayectorias de los estudiantes que se encuentran en situaciones de riesgo escolar. Así, se proponen diversas acciones relacionadas a: organizar propuestas de enseñanza interdisciplinar; generar propuestas sociocomunitarias; actividades complementarias. También se sugiere la alternativa de ofrecer Tutorías Académicas (clases de apoyo opcionales no curricularizadas) para aquellos estudiantes que necesitan recuperar aprendizajes, realizar acompañamiento, etc. Para llevar adelante esta tarea, el Estado Nacional transfiere fondos a las provincias para formar equipos técnicos territoriales $\mathrm{y}$, además, contratar profesores (tutores académicos) que se suman al equipo institucional y trabajan de forma articulada con los 


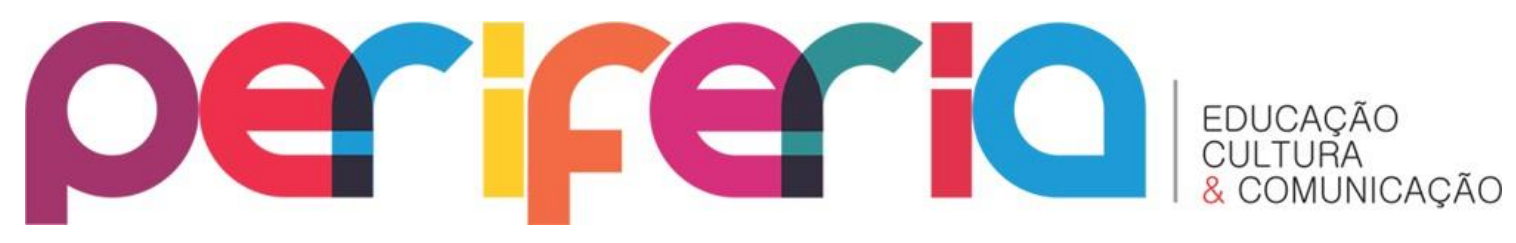

docentes de cátedra. El programa se inició en 2010 con el 50\% de las escuelas de todo el país, y en 2011 se extendió al resto.

En un trabajo de Terigi et.al (2013), se plasman algunas características interesantes del PMI y se afirma que el mismo presenta alguna potencialidad para promover cambios en la matriz organizacional tradicional ya que amplía el tiempo de ciertos profesores en la escuela permitiendo que se desempeñen nuevos roles (ej. Tutores); supone una posibilidad para ampliar el tiempo en la escuela por parte del estudiante; promueve la incorporación de nuevos docentes en la escuela aunque sea en modo transitorio; permite un trabajo más personalizado con los estudiantes que asisten a las clases de apoyo. No obstante, uno de los riesgos que señala se vincula a que la innovación que promueve el PMI opera en el marco de las condiciones de escolarización de la escuela secundaria estándar, y por esta razón es posible que se diluya el propósito transformador de la propuesta.

\section{URUGUAY: PROGRAMA DE IMPULSO A LA UNIVERSALIZACIÓN (PIU)}

En el año 2009, la Ley General de Educación N⒙437 establece la educación secundaria obligatoria. Así en el art. $7^{\circ}$ se describe: "Es obligatoria la educación inicial para los niños y niñas de cuatro y cinco años de edad, la educación primaria y la educación media básica y superior...”. Respecto de sus objetivos se plantea que la educación media básica abarca el ciclo inmediato posterior a la educación primaria; profundiza en el desarrollo de competencias y conocimientos adquiridos; y promueve el dominio teóricopráctico de diferentes disciplinas" (Art. 27). La educación media superior abarca hasta tres años posteriores a la educación media básica; tendrá tres modalidades cada una de las cuales ofrecerá mayor nivel de profundización y especialización: educación general (permite la continuidad en la educación terciaria), tecnológica (permite continuar estudios terciarios y la inserción laboral), técnica-profesional (orientada a la inserción laboral). La culminación de cualquiera de estas tres modalidades permitirá la continuación de estudios 


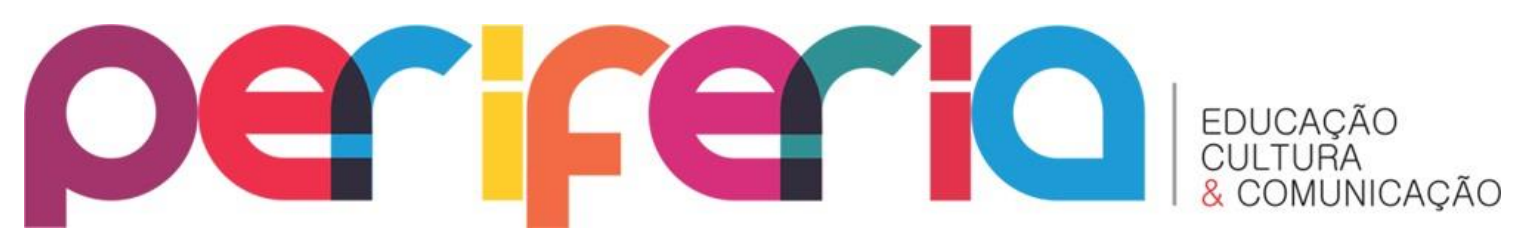

(Art. 28). Como en el caso argentino, la promulgación de una nueva ley de educación que establece la obligatoriedad del nivel medio, fue el puntapié inicial para el fomento de políticas específicas hacia los jóvenes en edad escolar. Entre ellos se pueden mencionar: Programa de Impulso a la Universalización (PIU), Programa de Aulas Comunitarias (PAC), Plan de Formación Profesional Básica (FPB).

El Programa de Impulso a la Universalización (PIU) tiene en las tutorías su dispositivo central ya que identifica los estudiantes con mayor riesgo de fracaso (unos 19.000) y les ofrece un espacio diferenciado y personalizado de grupos pequeños en el que se trabaja sobre sus dificultades y se los apoya en su desempeño escolar (ARISTIMUÑO, 2011). Se trata del principal programa con el que este país atiende al problema del fracaso escolar alcanzando en 2009, 78 liceos (37 en Montevideo y 41 en el resto del país). Sus principales destinatarios son aquellos adolescentes que asisten al ciclo básico de los liceos con mayores índices de abandono y repitencia. En este sentido, uno de sus principales objetivos radica en la mejora de los aprendizajes para el incremento de los índices de promoción. Son cinco los componentes sobre los cuáles se organiza el PIU: fortalecimiento técnico-pedagógico de los liceos (horas docentes); fortalecimiento de los equipos técnicos de atención psicosocial; transferencia de recursos de ejecución liceal (alimentación, útiles, transporte, vestimenta); apoyo a los alumnos vulnerables al fracaso (cuya actividad central son las tutorías); participación social y comunicación social (ARISTIMUÑO, 2011).

Fernández Aguerre y Alonso Bianco (2012) sostienen que la emergencia de este tipo de programas se vincula a la articulación lograda entre la innovación de la política con las instituciones de educación media del país. La misma se fundamenta en el imperativo actual de la inclusión educativa que se propone cumplir con dos grandes objetivos: fortalecer la integración a la escuela a jóvenes escolarizados que tienen un alto nivel de riesgo de abandonar; y revincular a quienes han abandonado la educación formal sin completar el nivel que estaban cursando (AINSCOW; MILES, 2008). Esto se 


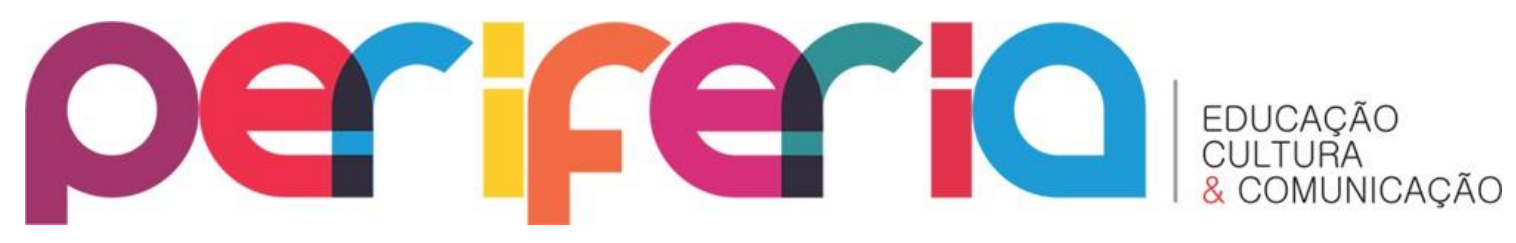

demuestra en la investigación realizada por Aristimuño (2011) donde se afirma que las tutorías parecen haberse instalado como un espacio que atiende a un problema real y concreto. Así, los estudiantes con mayor riesgo de abandono han encontrado un espacio que los ayuda a comprender con mayor perspectiva el rol de la educación visualizando nuevas formas de aproximarse al conocimiento y a las exigencias que plantea su pasaje por el liceo. Al mismo tiempo, los docentes afirman que las tutorías enriquecen el trabajo docente fortaleciendo el trabajo colectivo. Sin embargo, en la gestión del programa aparecen algunos inconvenientes relacionados al componente económico, ya que las partidas de dinero a veces se ven demoradas. Además, la gran flexibilidad que presenta el programa a la hora de diseñar los dispositivos institucionales, genera una gran diversidad y disparidad de experiencias que se traducen en resultados muy diversos.

\section{CHILE: LICEO PARA TODOS}

En el caso chileno, la Ley General de Educación $N^{\circ} 20.370$ promulgada en 2009 establece que “... la educación básica y la educación media son obligatorias, debiendo el Estado financiar un sistema gratuito destinado a asegurar el acceso a ellas de toda la población, así como generar las condiciones para la permanencia en el mismo de conformidad a la ley..." (Art.4). Además, define como principal finalidad procurar que cada alumno expanda y profundice su formación general y desarrolle los conocimientos, habilidades, actitudes que le permitan ejercer una ciudadanía activa e integrarse a la sociedad. Este nivel ofrece como formaciones diferenciadas: humanístico-científica; técnico-profesional y artística. Todas ellas habilitan al alumno a continuar su proceso educativo formal o incorporarse a la vida del trabajo (Art. 20).

Un dato no menor, es que en el período 1990-2010 (equivalente a cuatro gobiernos de una misma alianza de centro-izquierda) se desarrollan un conjunto de políticas públicas en educación que tuvieron un alto impacto en 


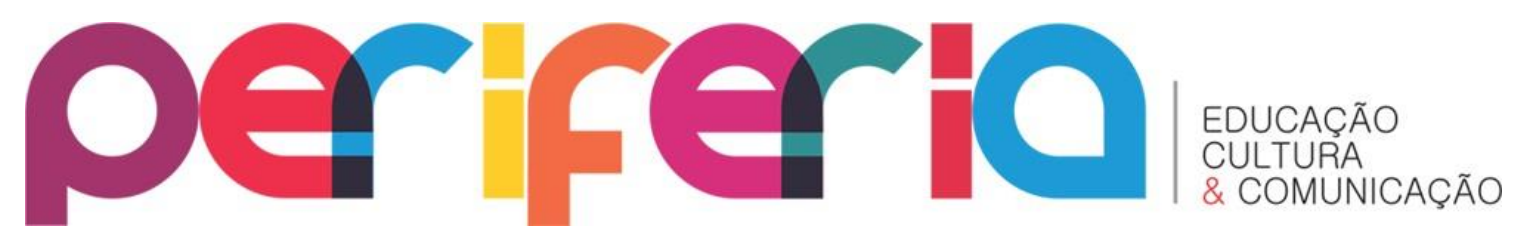

cuanto a cobertura, calidad y equidad del sistema escolar (COX, 2012). En medio de ese período, dichas políticas se institucionalizan y legitiman a través de la sanción de la Ley General de Educación (LGE). Podríamos decir, entonces, que el Programa Liceo para Todos creado en el año 2000 (a diferencia de los casos analizados previamente de Argentina y Uruguay) antecede a las regulaciones de LGE. El propósito fundamental de este programa es mejorar la calidad de los liceos que atienden a jóvenes de mayor vulnerabilidad socio-económica y educativa. Sus principales componentes son: a. becas para alumnos con riesgo de deserción; b. estrategias para mejorar las formas de mejorar la enseñanza; y c. apoyo a la iniciativas y planes de cada liceo para reducir la deserción y mejorar las oportunidades de aprendizaje (MARSHALL INFANTE, 2004). De este modo, se despliegan estrategias que apuntan a retener a los jóvenes en los liceos a la vez que se procura que su experiencia escolar sea significativa y habilitadora con el fin de insertarse en la sociedad con igualdad de oportunidades.

Respecto de su dimensión pedagógica, el programa Liceo Para Todos busca crear una forma de enseñanza que contemple caminos diversificados, frente a distintas disposiciones de aprendizaje. Para restituir aprendizajes no logrados de los estudiantes se desarrolla una acción pedagógica diferenciada en los primeros años de cada liceo, centrada en Lenguaje y Matemática. Se inicia con un diagnóstico que busca explicitar las competencias y disposiciones reales de aprendizaje de cada estudiante organizando a los mismos por grupo nivel. A partir de allí se proponen materiales y experiencias diferenciadas de aprendizaje de acuerdo a los puntos de partida de cada estudiante. Aunque el recorrido es diferenciado, el punto de llegada es común: los aprendizajes establecidos en el curriculum. Cabe destacar que, a diferencia de las clases de apoyo o tutorías de los programas analizados previamente (PMI en Argentina y PIU en Uruguay), la innovación se instala en la sala de clases. No se trata de clases adicionales para alumnos con dificultades, sino de reorganizar las clases regulares de Lenguaje y Matemática de todos los alumnos, a partir de una profunda comprensión de la estructura del 


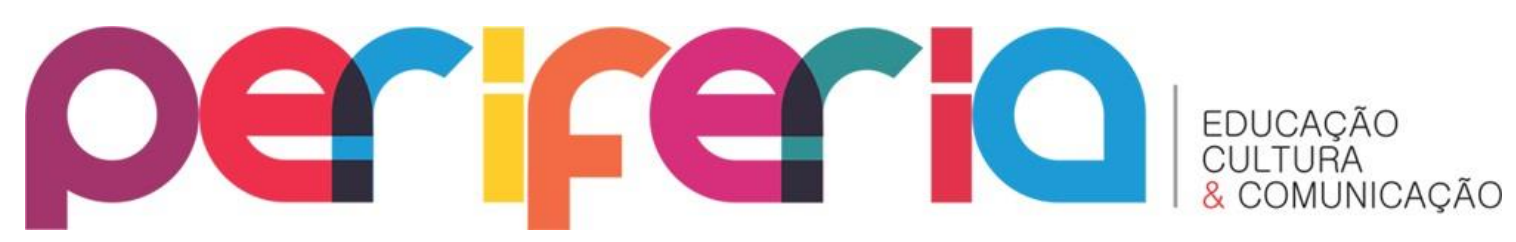

curriculum por parte del profesor y de un conocimiento detallado de los aprendizajes de los alumnos (JACINTO; TERIGI, 2007).

\section{CRUCE DE DATOS Y ANÁLISIS COMPARATIVO}

Casimiro Lopes, Rodriguez da Cunha y Costa (2013) proponen la noción de traducción como categoría de análisis permite comprender los juegos del lenguaje en campos discursivos sin fijar una posible significación. Analizar las contingencias es una forma de escapar a los límites que la propia definición impone. Explorar las condiciones que favorecen una interpretación contextual, sin establecer que sus sentidos son deudores de una lectura previa obligatoria, puede ser productivo para el entendimiento de los procesos de traducción. Los límites de un imaginario socio-político que hegemoniza ciertos discursos no bloquean las posibilidades de que nuevos sentidos sean diseminados. La traducción, entonces, es entendida como apertura a la alteridad, o de otro modo, como imposibilidad de resistencia a la alteridad

A continuación, se presenta una tabla comparativa donde se destacan las principales características que asumen, en cada país, las políticas educativas desarrolladas para la educación secundaria donde se vislumbran estos procesos de traducción. 


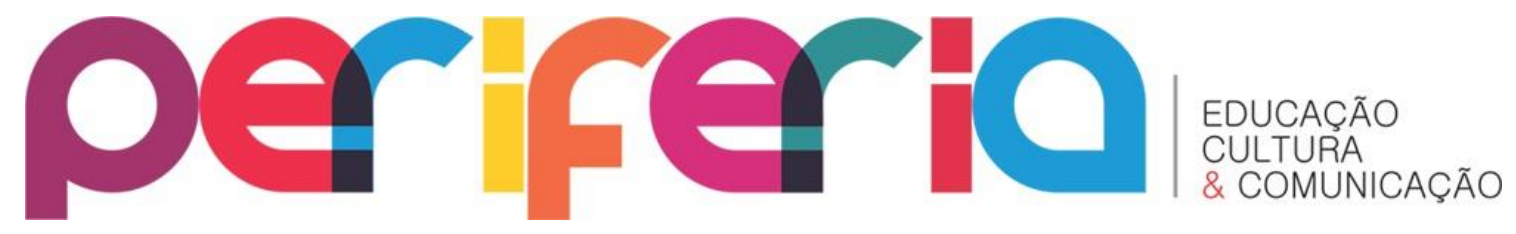

Cuadro 1: Políticas públicas para la Educación secundaria: análisis comparativo.

\begin{tabular}{|c|c|c|c|c|}
\hline $\begin{array}{l}\text { Políticas } \\
\text { Públicas } \\
\text { para la } \\
\text { E.S }\end{array}$ & Dimensiones & Argentina & Uruguay & Chile \\
\hline \multirow{4}{*}{ 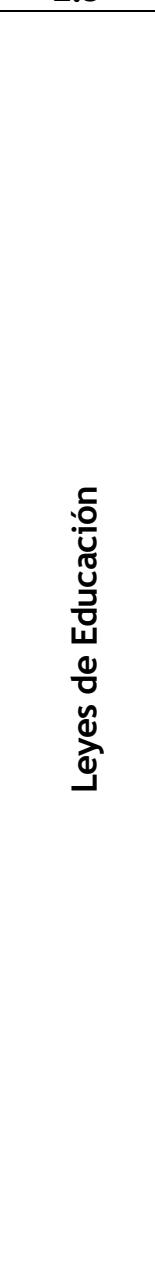 } & $\begin{array}{l}\text { Denominación y } \\
\text { año de sanción }\end{array}$ & $\begin{array}{l}\text { Ley de Educación } \\
\text { Nacional } \\
26.206 / 2006 \text {. }\end{array}$ & $\begin{array}{c}\text { Ley General de } \\
\text { Educación } \\
18.437 / 2009\end{array}$ & $\begin{array}{c}\text { Ley General de } \\
\text { Educación 20.370/2009 }\end{array}$ \\
\hline & Obligatoriedad & $\begin{array}{l}\text { Establece la } \\
\text { obligatoriedad de } \\
\text { la educación } \\
\text { secundaria. }\end{array}$ & $\begin{array}{c}\text { Establece la } \\
\text { obligatoriedad } \\
\text { de la educación } \\
\text { secundaria. }\end{array}$ & $\begin{array}{c}\text { Establece la } \\
\text { obligatoriedad de la } \\
\text { educación secundaria. }\end{array}$ \\
\hline & Objetivos & $\begin{array}{l}\text { Formación para la } \\
\text { ciudadanía, el } \\
\text { trabajo y la } \\
\text { continuidad de } \\
\text { estudios } \\
\text { superiores. }\end{array}$ & $\begin{array}{l}\text { Profundiza en el } \\
\text { desarrollo de } \\
\text { competencias y } \\
\text { conocimientos } \\
\text { adquiridos; y } \\
\text { promueve el } \\
\text { dominio teórico- } \\
\text { práctico de } \\
\text { diferentes } \\
\text { disciplinas. }\end{array}$ & $\begin{array}{l}\text { Procurar que cada } \\
\text { alumno expanda y } \\
\text { profundice su formación } \\
\text { general y desarrolle los } \\
\text { conocimientos, } \\
\text { habilidades, actitudes } \\
\text { que le permitan ejercer } \\
\text { una ciudadanía activa e } \\
\text { integrarse a la sociedad }\end{array}$ \\
\hline & $\begin{array}{l}\text { Orientaciones/M } \\
\text { odalidades }\end{array}$ & $\begin{array}{c}10 \text { orientaciones } \\
\text { (agro y ambiente, } \\
\text { ciencias sociales, } \\
\text { cs. soc. y } \\
\text { humanidades, } \\
\text { ciencias naturales, } \\
\text { comunicación, } \\
\text { arte, informática, } \\
\text { economía y } \\
\text { administración, } \\
\text { turismo, } \\
\text { educación física, } \\
\text { lenguas } \\
\text { extranjeras). }\end{array}$ & $\begin{array}{l}3 \text { modalidades } \\
\text { (general, } \\
\text { tecnológica y } \\
\text { técnica- } \\
\text { profesional) }\end{array}$ & $\begin{array}{c}3 \text { modalidades } \\
\text { (humanístico-científica; } \\
\text { técnico-profesional y } \\
\text { artística) }\end{array}$ \\
\hline \multirow{4}{*}{ 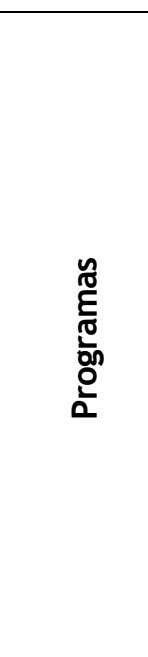 } & Denominación & PMI & PIU & Liceo Para Todos \\
\hline & Año de Inicio & 2010 & 2009 & 2000 \\
\hline & $\begin{array}{l}\text { Objetivo } \\
\text { principal }\end{array}$ & $\begin{array}{l}\text { Acompañar a las } \\
\text { trayectorias de los } \\
\text { estudiantes en } \\
\text { riesgo escolar. }\end{array}$ & $\begin{array}{l}\text { Mejora de los } \\
\text { aprendizajes } \\
\text { para el } \\
\text { incremento de } \\
\text { los índices de } \\
\text { promoción. }\end{array}$ & $\begin{array}{l}\text { Mejorar la calidad de los } \\
\text { liceos que atienden a } \\
\text { jóvenes de mayor } \\
\text { vulnerabilidad. }\end{array}$ \\
\hline & Componentes & $\begin{array}{l}\text {-Transferencia de } \\
\text { recursos a las } \\
\text { provincias y a las } \\
\text { escuelas. } \\
\text {-Tutorías } \\
\text { Académicas. }\end{array}$ & $\begin{array}{l}\text {-Tutorías como } \\
\text { dispositivo } \\
\text { central. } \\
\text {-Fortalecimiento } \\
\text { técnico- } \\
\text { pedagógico. } \\
\text {-Transferencia } \\
\text { de recursos. }\end{array}$ & $\begin{array}{l}\text {-Becas para alumnos con } \\
\text { riesgo de deserción. } \\
\text {-Estrategias para } \\
\text { mejorar las formas de } \\
\text { mejorar la enseñanza. } \\
\text {-Apoyo a las iniciativas y } \\
\text { planes de cada liceo. }\end{array}$ \\
\hline
\end{tabular}

Fuente: elaboración propia (2017) 


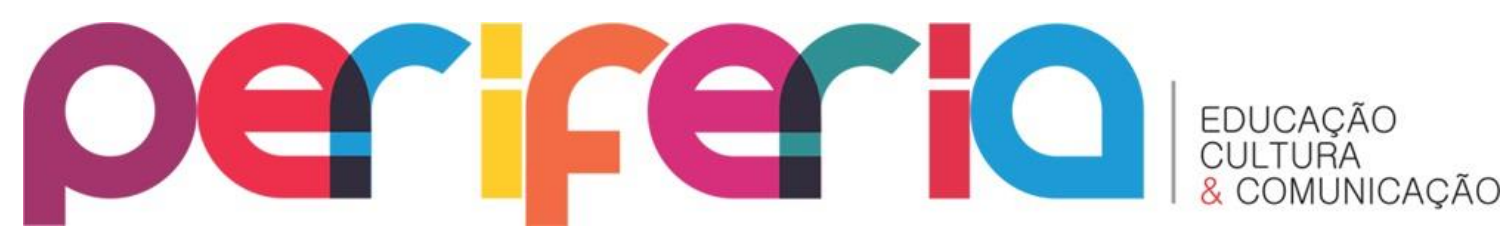

Como se puede observar, en rasgos generales los tres países analizados presentan algunas similitudes, aunque Argentina y Uruguay tienen mayores coincidencias respecto del desarrollo de políticas públicas y programas para la educación secundaria. Es importante mencionar que los tres países, tal como se dijo al principio de este trabajo, establecen la obligatoriedad de la educación secundaria a partir de las leyes de educación vigente. Argentina difiere respecto de Uruguay y Chile en relación al tránsito por el ciclo orientado (denominado en los otros dos países como ciclo superior) ya que ofrece diez orientaciones y aquellos sólo tres modalidades. Se podría afirmar entonces que en Argentina la oferta es más diversificada. Algo que comparten los tres países es que más allá de las orientaciones o modalidades que cursen los estudiantes, todas habilitan a continuar estudios superiores, o bien, ingresar al mundo laboral. La formación ciudadana también aparece como un rasgo importante en la formación.

Otro punto importante a tener en cuenta es que, en el caso de Argentina y Uruguay, las leyes vigentes anteceden a los programas analizados (PMI, y PIU respectivamente) mientras que en Chile con la puesta en marcha del Liceo para Todos se da el proceso inverso, siendo este precedente a la ley vigente. Esto evidencia la potencia de la traducción como mecanismo que opera en los diferentes contextos donde las políticas educativas se desarrollan.

Cabe destacar que los objetivos a los que responden cada uno de los programas son similares ya que los tres se dirigen a la mejora de la calidad de los aprendizajes y el incremento de la promoción atendiendo a jóvenes con cierto riesgo de abandono escolar. También los componentes de los programas evidencian algunas coincidencias en dos aspectos fundamentales: la transferencia de recursos a las escuelas, y el eje puesto en la institución como promotora de la gestión y mejora de los aprendizajes. No obstante, Uruguay y Argentina presentan mayores coincidencias respecto del formato Tutoría como la clave de la retención y contención de los jóvenes en la escuela, mientras 


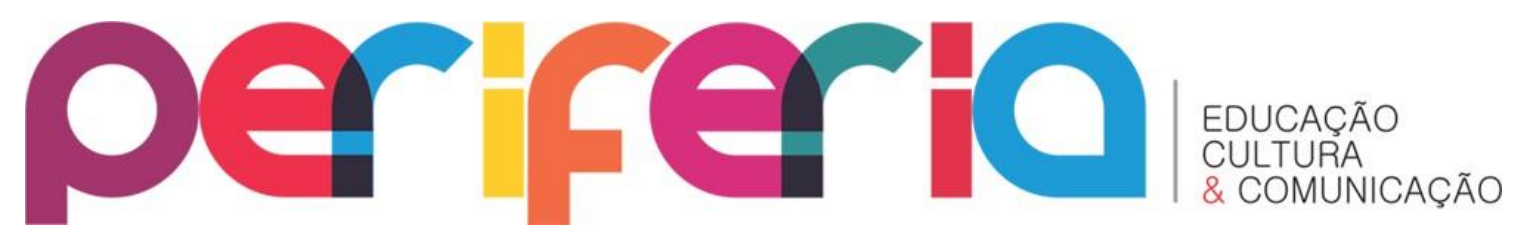

que en Chile la innovación se da dentro de la sala de clase con el profesor de cátedra.

En un trabajo elaborado por Jacinto y Terigi para la UNESCO (2006), se releva una serie de estrategias que las instituciones educativas desarrollan en pos de mejorar los índices de permanencia y promoción de la educación secundaria en América Latina. Así las autoras mencionan estrategias centradas en: el ausentismo de los alumnos, sobreedad de los estudiantes, reformulación de los tiempos de instrucción, reformulación del régimen académico, convivencia en las escuelas, tutorías y otras acciones de orientación a los estudiantes, compensación de los aprendizajes, reformulación didáctica de las clases presenciales, formación para el trabajo. Respecto de las tutorías se señala que un buen desarrollo del agente que cumple con la función del tutor requiere del marco de un proyecto institucional de tutoría que involucre a los profesores de cada curso, el equipo directivo de la escuela, los profesionales de apoyo (asesor pedagógico, psicólogo, otros) cuando los hay, etc. Una de las principales limitaciones de la acción de tutoría se presenta cuando el proyecto de tutoría queda restringido a las tareas del tutor.

Podemos afirmar que, más allá de las características particulares que en cada territorio asumen estas políticas, las tres comparten el propósito de acompañar a las trayectorias de los estudiantes y, ese acompañamiento, se desarrolla con una fuerte impronta institucional que intenta desafiar los núcleos más duros del formato escolar de la escuela secundaria. Ahora bien, cabría preguntarnos: ¿Es suficiente contar con recursos económicos y tiempos de extensión en la escuela, tanto para docentes como para estudiantes, para dislocar el formato escolar? ¿Hasta qué punto las tutorías tocan el núcleo duro de la organización institucional de la educación secundaria? ¿Cuáles son los modos a través de los cuáles los dispositivos que se ponen en marcha en los programas analizados promueven cambios estructurales al interior de las escuelas secundarias? ¿De qué manera logran estos programas desafiar el trípode de hierro del patrón organizacional propio de la educación secundaria? 


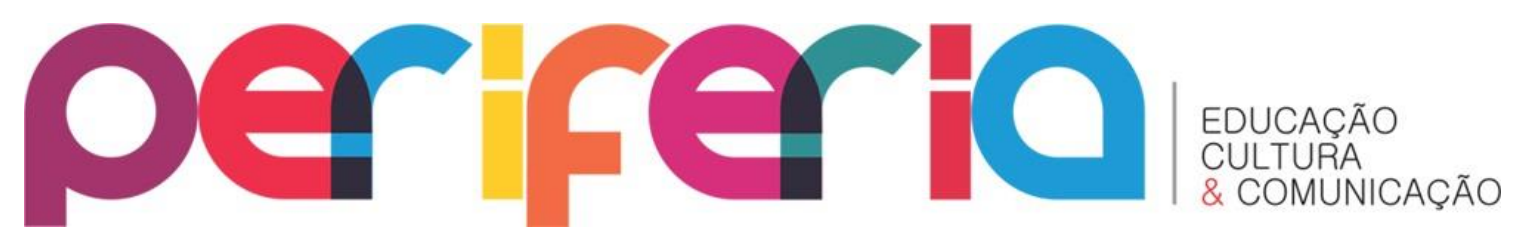

¿Cómo se juega la tensión entre lo local y lo global en estos aspectos? ¿Cuáles son las traducciones que se juegan al interior de cada micro nivel institucional? Estos y muchos otros interrogantes que al lector le surjan, operan como motor para seguir indagando, profundizando y reflexionando hacia nuevas formas de pensar, organizar y transitar la educación secundaria.

\section{A MODO DE REFLEXIÓN}

El presente artículo pone sobre la mesa los embates que en la actualidad enfrenta la educación secundaria en tres países de América Latina. A través de un estudio comparado en clave de traducción local/global, se vislumbra que la intención de generar cambios en su formato con el fin de lograr que todos/as los/as jóvenes ingresen, permanezcan, aprendan y egresen de la escuela secundaria, supera los límites de los Estados nacionales. Esto significa, que no podemos desconocer el contexto latinoamericano y mundial en el que estos cambios tienen lugar. La lectura desde el nivel supra hacia el nano, enriquece el análisis mostrando las tensiones y traducciones que se dan entre las políticas globales y locales. Así, en el supra nivel la Oficina Regional de la UNESCO para América Latina y El Caribe creó el Foro y Observatorio de Educación Secundaria cuyo objetivo primordial son los intercambios, la producción de conocimientos y el diálogo abierto y permanente para llevar adelante un trabajo conjunto y permanente en pos de transformar y mejorar la educación secundaria en la región (UNESCO, 2005). En este marco, se señala que desde finales del siglo XX y principios del siglo XXI los organismos internacionales generaron diversidad de iniciativas y compromisos internacionales vinculados con la educación secundaria en América Latina y el Caribe de los cuales se desprenden dos grandes áreas de preocupación: lograr la equidad en la educación secundaria y la formación integral de los adolescentes y jóvenes. En este sentido, los gobiernos latinoamericanos y caribeños se comprometieron a lograr porcentajes cada vez mayores de jóvenes que terminen la escuela secundaria. 


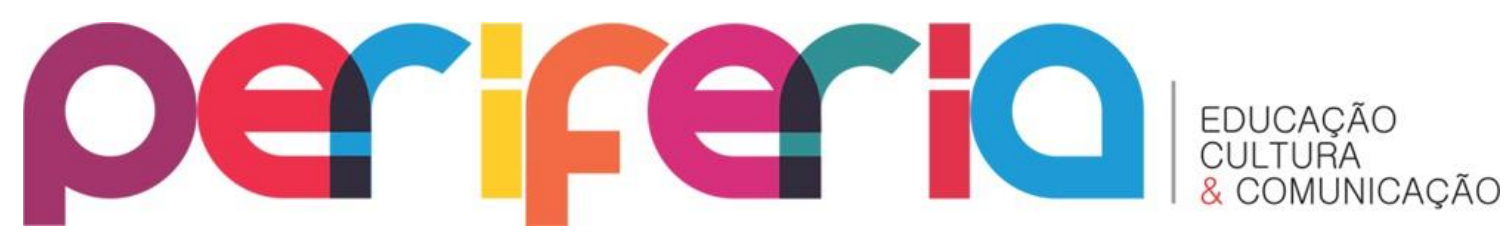

Optamos por realizar un estudio comparado entre Argentina, Uruguay y Chile con el fin de analizar las políticas educativas que estos países desarrollan analizando normativas y programas específicos. A partir de ello, pudimos vislumbrar características comunes y particulares que dan cuenta de las tensiones permanentes entre lo local y lo global (BALL, 2001) donde el segundo no puede destruir al primero debido a que cada contexto local es particular.

Para abordar esta tensión, Casimiro Lopes, Rodriguez da Cunha y Costa (2013) desarrollan dos formas de análisis. Por un lado, analizan la recontextualización acudiendo a los aportes de Ball (2001), donde opera la idea que hay una reinterpretación, una fluctuación de sentidos y un deslizamiento de interpretaciones de un contexto a otro. Hay una interpretación estabilizada de un fundamento original que se transforma en otra interpretación estabilizada por el proceso de transferencia físico/simbólico, que no llega a constituir una desestructuración o un cambio del centro de la estructura. En cambio, las autoras proponen superar la noción de recontextualización abordando el análisis desde una noción de traducción donde se comprometen los juegos del lenguaje. En la traducción, la circulación del significante no tiene origen ni tiene fin, es un proceso incesante.

Argentina, Uruguay y Chile se presentan entonces como contextos macro de políticas educativas que dialogan con el nivel supra, en este caso la UNESCO, a la vez que entran en tensión con los niveles micro institucionales donde cada escuela y cada profesor en el nano nivel, despliega una serie de dispositivos y estrategias particulares para materializar en acciones concretas las políticas educativas que atienden a la educación secundaria.

¿Cuál es el alcance de esos dispositivos y estrategias? ¿De qué modo garantizan la obligatoriedad de la educación secundaria? ¿Generan reales acciones de inclusión estos programas? Son algunas de las preguntas que quedan abiertas, para continuar indagando y analizando los sentidos que la educación secundaria pueda construir en el presente siglo. 


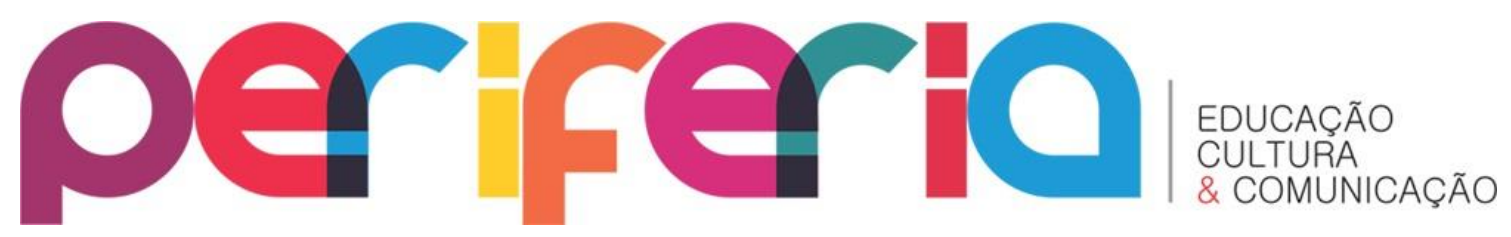

\section{BIBLIOGRAFÍA}

ACOSTA, Felicitas. La escuela secundaria argentina en perspectiva histórica y comparada: modelos institucionales y desgranamiento durante el siglo XX. Cuadernos de historia de la educación, v. 11, n. 1, p. 131-144, 2012.

AINSCOW, Mel; MILES, Susi. Por una educación para todos que sea inclusiva: ¿hacia dónde vamos ahora. Perspectivas, Paris, v. 38, n. 1, p.17-44, mar. 2008.

ALTBACH, Philip; KELLY, Gail. Nuevos enfoques en educación comparada. Madrid: Mondadori, 1990.

ARGENTINA. Ley $N^{\circ}$ 26.206/2006 de Educación Nacional.

ARGENTINA. Resolución CFE 84/09 "Lineamientos Políticos y Estratégicos de la Educación Secundaria Obligatoria”.

ARGENTINA. Resolución CFE 88/09 "Institucionalidad y Fortalecimiento de la Educación Secundaria Obligatoria: Planes Jurisdiccionales y Planes de Mejora Institucional (PMI)".

ARGENTINA. Resolución CFE 93/09 "Orientaciones para la organización pedagógica e institucional de la Escuela Secundaria Obligatoria".

ARGENTINA. Resolución CFE 103/10 "Pautas Federales para la movilidad estudiantil en la Educación Obligatoria”.

ARGENTINA. Resolución CFE 102/10 "Propuestas de inclusión y/o regularización de trayectorias escolares en la educación secundaria"

ARISTIMUÑO, Adriana. La inclusión como objetivo ante el fracaso escolar en la Secundaria básica de Uruguay. Revista latinoamericana de educación inclusiva, v. 5, n. 2, p. 59-81, 2011.

BENAVOT, Aaron. La diversificación en la educación secundaria: Currículos escolares desde la perspectiva comparada. Profesorado. Revista de curriculum y formación del profesorado, ano 1, n. 10, p. 1-30, 2006.

BENTANCUR, Nicolás. ¿Hacia un nuevo paradigma en las políticas educativas? Las reformas de las reformas en Argentina, Chile y Uruguay (2005-2007). Revista Uruguaya de Ciencia Política, Montevideo, v. 16, n. 1, p. 159-179, 2007.

BRAY, Mark; ADAMSON, Bob; MASON, Mark. Educación comparada, enfoques y métodos. Buenos Aires: Granica, 2010. 


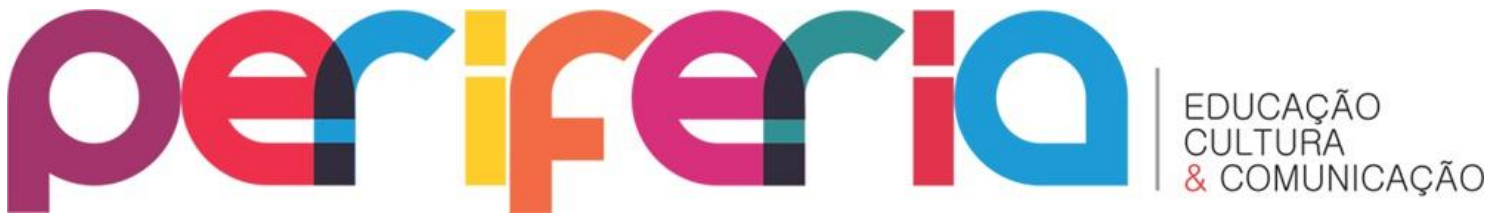

CASIMIRO LOPES, Alice; VIRGILIO RODRIGUEZ DA CUNHA, Erika; COSTA, Hugo. Da recontextualização à tradução: investigando políticas de currículo. Currículo sem Fronteiras, v.13, n. 3, p. 392-410, set./dez. 2013.

CHILE. Ley N²0.370/2009 General de Educación.

COX, Cristian. Política y políticas educacionales en Chile 1990-2010. Revista Uruguaya de Ciencia Política, Montevideo, v. 21, n. 1, p. 13-42, jan./jun. 2012.

FERNÁNDEZ AGUERRE, Tabaré y ALONSO BIANCO, Cecilia. Dos modelos de inclusión educativa: programa de aulas Comunitarias y plan de formación profesional básica en Uruguay (2007-2011). Revista Uruguaya de Ciencia Política, Montevideo, v. 21, n. 1, p. 161-182, jan./jun. 2012.

GOROSTIAGA, Jorge. Las políticas para el nivel secundario en Argentina: ¿Hacia una educación más igualitaria? Revista Uruguaya de Ciencia Política, Montevideo, v. 21, n. 1, p. 141-159, jan./jun. 2012.

GUREVICH, Raquel. Educar en tiempos contemporáneos: una práctica social situada. Revista Propuesta Educativa, n. 32, ano 18, p. 23-31, nov. 2009.

JACINTO, Claudia. Consideraciones sobre estrategias de inclusión con calidad en la escuela secundaria. Debate N. 7. Universalizar el acceso y completar la educación secundaria. Entre la meta social y la realidad Latinoamericana, p. 29-38, nov. 2010.

JACINTO, Claudia y TERIGI, Flavia. ¿Qué hacer ante las desigualdades en la educación secundaria?: aportes de la experiencia latinoamericana. Buenos Aires: UNESCO-IIPE/Santillana, 2007.

MARSHALL INFANTE, María Teresa. Programas de mejoramiento de las oportunidades. El Liceo para Todos en Chile. Instituto internacional de planeamiento para la educación/UNESCO, 2004.

MINISTERIO DE EDUCACIÓN DE LA NACIÓN. La política educativa nacional. Inclusión y mejores aprendizajes para la igualdad educativa. 2003-2015. Buenos Aires, Argentina, 2015.

OFICINA REGIONAL DE EDUCACIÓN PARA AMÉRICA LATINA Y EL CARIBE, UNESCO. Situación educativa de América Latina y El Caribe: hacia la educación de calidad para todos al 2015. Santiago de Chile: Ediciones del Imbunche, 2013.

OFICINA REGIONAL DE EDUCACIÓN PARA AMÉRICA LATINA Y EL CARIBE, UNESCO. Educación secundaria en la región: ¿Dónde estamos y hacia dónde vamos? UNESCO, 2005. 


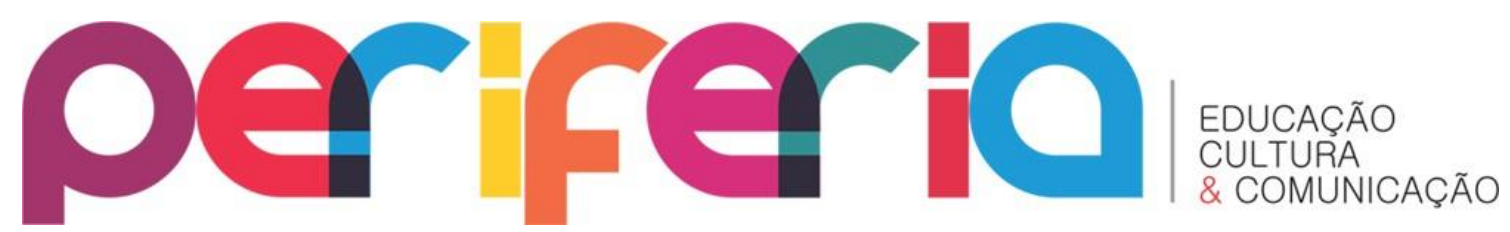

PINEAU, Pablo; SOUTHWELL, Miriam. Clase 1. La constitución histórica de la Educación Secundaria en Europa y América Latina. Seminario: La educación secundaria: principales temas y problemas en perspectiva latinoamericana. FLACSO, 2010.

PINKASZ, Daniel. Sobre la escuela como eje del cambio de las políticas de educación secundaria en la Argentina. Revista Propuesta Educativa, n. 44, ano 24, p. 8-23, nov. 2015.

SÁNCHEZ TEPATZI, José de la Luz. ¿Cómo se traduce el curriculum formal en una experiencia formal relevante para los alumnos de una escuela telesecundaria de México? Actualidades investigativas en educación, San Pedro de Montes de Oca, Costa Rica, v. 11, n. 2, p. 1-22, 2011.

SANTOS GUERRA, Miguel Ángel. El proyecto de centro: Una tarea comunitaria, un proyecto de viaje compartido. Em: GIMENO SACRISTÁN, José. Saberes e incertidumbres sobre el curriculum. Madrid: Morata, 2010.

STIRLING NETWORK FOR CURRICULUM STUDIES. Disponible en: http://www.stir.ac.uk/social-

sciences/research/ourresearchcentresandnetworks/stirlingnetworkforcurricul umstudies/ (Consultado 27 de mayo de 2017).

TERIGI, Flavia. Los cambios en el formato de la escuela secundaria argentina: por qué son necesarios, por qué son tan difíciles. Revista Propuesta Educativa, v. 1, n. 29, ano 15, p. 63-71, 2008.

TERIGI, Flavia; BRISCIOLI, Bárbara; SCAVINO, Carolina; MORRONE, Aldana y TOSCANO, Ana Gracia. La educación secundaria obligatoria en Argentina: entre la expansión del modelo tradicional y las alternativas de baja escala. Dossier La educación secundaria en la Argentina. Revista del IICE, n. 33, p. 27-46, 2013.

URUGUAI. Ley N 18.437/2009 General de Educación. 